\title{
Traduction et adaptation en langue française du Lasater Clinical Judgment Rubric: Une étude multicentrique
}

Jacinthe Beauchamp

Centre de formation médicale du Nouveau-Brunswick, jacinthe.beauchamp@umoncton.ca

Michelle Lalonde

Université d'Ottawa, michelle.lalonde@uottawa.ca

Viviane Fournier

Université du Québec en Abitibi-Témiscamingue, Viviane.fournier@uqat.ca

Sabrina Mehiz

Haute Ecole Arc Santé, Haute Ecole Spécialisée de Suisse Occidentale HES-SO, Sabrina-mehiz@he-arc.ch

Marco Pedrotti

Haute Ecole Arc Santé, Haute Ecole Spécialisée de Suisse Occidentale HES-SO, marco.pedrotti@he-arc.ch

Isabelle Michel

Collège Boréal, isabelle.michel@collegeboreal.ca

Pierre Godbout

Université de Moncton, Bathurst, pierre.godbout@umoncton.ca

Ivan Simoneau

Cegep de Sherbrooke, ivan.simoneau@cegepsherbrooke.qc.ca

See next page for additional authors

Follow this and additional works at: https://qane-afı.casn.ca/journal

Part of the Higher Education Commons, and the Nursing Commons

\section{Recommended Citation}

Beauchamp, Jacinthe; Lalonde, Michelle; Fournier, Viviane; Mehiz, Sabrina; Pedrotti, Marco; Michel, Isabelle; Godbout, Pierre; Simoneau, Ivan; and Lasater, Kathie (2021) "Traduction et adaptation en langue française du Lasater Clinical Judgment Rubric: Une étude multicentrique," Quality Advancement in Nursing Education - Avancées en formation infirmière: Vol. 7: Iss. 2, Article 5.

DOI: https://doi.org/10.17483/2368-6669.1277

This Article is brought to you for free and open access by Quality Advancement in Nursing Education - Avancées en formation infirmière. It has been accepted for inclusion in Quality Advancement in Nursing Education - Avancées en formation infirmière by an authorized editor of Quality Advancement in Nursing Education - Avancées en formation infirmière. 


\section{Traduction et adaptation en langue française du Lasater Clinical Judgment Rubric: Une étude multicentrique}

\section{Cover Page Footnote}

L'équipe de chercheurs remercie les participantes et les étudiantes qui ont participé aux vidéos, sans qui ce projet n'aurait pas été possible. Nous sommes aussi reconnaissants envers le Bureau de recherche du Centre de formation médicale du Nouveau-Brunswick, au Réseau-action Formation et Recherche de la Société Santé et Mieux-être en français du Nouveau-Brunswick et à la Haute École Spécialisée de Suisse Occidentale HES-SO pour leur appui financier. The research team would like to thank the participants and students who took part in the videos; this project would not have been possible without them. We are also grateful to the research office at the Centre de formation médicale du Nouveau-Brunswick, to the Réseauaction Formation et Recherche de la Société Santé et Mieux-être en français du Nouveau-Brunswick, and to the Haute École Spécialisée de Suisse Occidentale HES-SO for their financial support.

\section{Authors}

Jacinthe Beauchamp, Michelle Lalonde, Viviane Fournier, Sabrina Mehiz, Marco Pedrotti, Isabelle Michel, Pierre Godbout, Ivan Simoneau, and Kathie Lasater 


\section{Introduction}

Exercer un jugement clinique infirmier est considéré une compétence clé de la pratique infirmière, particulièrement lors de situations incertaines telles que la détérioration de la santé d'un patient (Lavoie et al., 2015). La pratique en milieu de soins aigus et critiques exige des infirmières ${ }^{1}$ un niveau élevé de cette compétence. Pourtant, des études soulignent, chez la plupart des infirmières nouvellement diplômées, un déficit quant au niveau du jugement clinique requis pour prendre en charge des situations complexes, c'est-à-dire des situations imprévisibles et instables (Kavanagh et Szweda, 2017; Lasater et al., 2015; Monagle et al., 2018; Parker et al., 2014).

\section{Contexte}

À partir d'une recension des écrits, Tanner (2006) a proposé un modèle du jugement clinique infirmier qu'elle définit comme « une interprétation ou une conclusion à propos des besoins, préoccupations ou problèmes de santé d'un patient et la décision d'entreprendre ou non une action... selon ce qui semble approprié en vertu de la réponse du patient » (traduction libre p. 204 par Lavoie et al., 2017). Ce modèle a d'ailleurs été récemment traduit et adapté en français (Lavoie et al., 2021). Il a également inspiré les travaux de Lasater (2007a) qui a développé une grille permettant l'appréciation du développement du jugement clinique, le Lasater Clinical Judgment Rubric. Une rubrique ou une grille est un instrument qui décrit une compétence et distingue son degré de maîtrise (Huba et Freed, 2000; Lesmond et al., 2017; Tardif, 2006).

À un moment où de plus en plus d'établissements d'enseignement supérieur intègrent l'apprentissage par simulation de situations de soins comme activité complémentaire aux stages en milieux cliniques (Deschênes et al., 2016), l'accès à ce type d'outil est précieux. Il fournit à l'apprenante l'information nécessaire pour s'améliorer. À notre connaissance, peu d'instruments validés et standardisés permettant d'apprécier le développement de certaines compétences telles que le jugement clinique sont disponibles en langue française. Ces instruments sont aussi nécessaires pour évaluer l'efficacité pédagogique des activités de simulation.

La traduction en langue française d'outils développés et déjà validés en langue anglaise représente une option efficace et efficiente. Elle offre l'avantage de profiter des connaissances obtenues par des recherches effectuées avec la version originale de l'outil et de permettre des comparaisons de nature internationale (Haccoun, 1987; Hébert et al., 1994; Sousa et Rojjanasrirat, 2011; Vallerand, 1989). À l'instar de plusieurs outils cliniques et éducatifs traduits et adaptés (Cloutier et al., 2015; Côté et al., 2018), la validation transculturelle permet également une utilisation plus uniforme de l'outil traduit entre régions ou pays utilisant une même langue, en neutralisant l'aspect culturel de la sémantique (Vallerand, 1989). Il est toutefois important de noter que des différences culturelles existent à plusieurs niveaux. La langue, les pratiques et les valeurs varient à l'intérieur d'une même province comme le Nouveau-Brunswick ou le Québec, entre provinces et entre pays. Outre la culture d'une région, il y a celle de l'établissement, du programme, et du rôle professionnel de l'infirmière. Le but de cette étude multicentrique internationale était de produire un instrument, fondé sur les résultats de la traduction et de la validation dans différentes régions de la francophonie d'un instrument existant, permettant d'apprécier le développement du jugement clinique.

\footnotetext{
${ }^{1}$ L'emploi du genre féminin a pour seul but d'alléger le texte et d'en faciliter la lecture. Le terme « infirmières » inclut les infirmières et les infirmiers.
} 


\section{Méthode}

Le processus de la traduction et de la validation transculturelle d'un instrument doit suivre des règles précises et rigoureuses afin d'assurer l'équivalence entre l'instrument source et celui traduit (Hébert et al., 1994; Sousa et Rojjanasrirat, 2011; Vallerand, 1989). En d'autres mots, cela permet de trouver un terrain commun par rapport à une nomenclature applicable dans différentes régions, dans notre cas la francophonie. La méthode de traduction et de validation proposée par Hébert, Bravo et Voyer (1994) a été retenue dans le cadre de cette étude. Cette méthode a guidé la traduction et la validation en langue française d'autres instruments utilisés dans le cadre d'études sur la simulation clinique en formation infirmière (Simoneau et Paquette, 2014; Simoneau et al., 2012). Elle consiste en cinq étapes : 1. la sélection d'un instrument valide et fidèle en langue anglaise; 2 . la traduction renversée; 3. la révision de l'instrument traduit en comité; 4. un prétest de l'instrument traduit, et 5 . une étude de fidélité test-retest.

\section{Étape 1 : Sélection de l'instrument}

Les travaux conduits par Adamson, Kardong-Edgren et Willhaus (2013) et par KardongEdgren, Adamson et Fitzgerald (2010) ont permis de dénombrer et d'inventorier plusieurs instruments de mesure de différentes dimensions se rapportant à l'efficacité de la simulation. Tous sont en langue anglaise et plus de la moitié portent sur le jugement clinique, ses composantes (p. ex. travail en équipe, évaluation, communication) ou ses variantes (p. ex. pensée critique, raisonnement clinique.) Parmi ces derniers, le Lasater Clinical Judgment Rubric (LCJR) s'est avéré un instrument de mesure approprié pour apprécier le développement du jugement clinique dans des contextes de formation par simulation (Adamson et al., 2012; Cato et al., 2009; Lasater, 2007a, 2007b, 2011; Shin et al., 2015; Sideras, 2007). Basé sur le modèle du jugement clinique de Tanner (2006) et à partir de données empiriques recueillies lors de simulations, Lasater (2007a) a conçu une grille décrivant le jugement clinique en différents niveaux de performance : beginning, developing, accomplished et exemplary. Une grille est utile autant pour l'enseignante que pour l'étudiante (Arter et Chappuis, 2006). D'une part, elle définit de façon transparente les cibles d'apprentissages complexes et des attentes et assure une appréciation cohérente dans le temps, entre activités d'apprentissage et entre enseignantes. D'autre part, elle oriente les étudiantes pour qu'elles puissent comprendre les attentes à différents moments de leur formation. Outre la pertinence des aspects du jugement clinique qu'il permet d'étudier (Tanner, 2006; Lasater, 2011), le LCJR possède aussi de bonnes propriétés métrologiques (Adamson et al., 2012; Davis et Kimble, 2011; Mariani et al., 2013; Shin et al., 2015). Utilisé dans de nombreuses recherches, le LCJR a montré des coefficients de cohérence interne ( $\alpha$ de Cronbach) entre 0,80 et 0,97 (Adamson et al., 2012; Mariani et al., 2013; Shin et al., 2015). L'instrument est déjà disponible dans d'autres langues dont le chinois (Yang et al., 2019), l'espagnol (Román-Cereto et al., 2018), le néerlandais (Vreugdenhil et Spek, 2018), le portugais brésilien (Nunes et al., 2016), le suédois (Kristiansen et al., 2015) et le coréen (Shin et al., 2015).

La grille LCJR a été conçue pour apprécier la trajectoire du développement du jugement clinique de l'étudiante selon une perspective longitudinale, au travers de son cheminement dans un programme de formation. La grille aborde quatre aspects du jugement clinique : remarquer (noticing), interpréter (interpreting), intervenir (responding), et réfléchir (reflecting). Ces aspects trouvent leurs sources dans les fondements du cadre conceptuel du Tanner Clinical Judgment Model (Tanner, 2006). La grille comprend 11 indicateurs de performance clinique (Clinical Performance Indicators) : 3 pour l'aspect remarquer, 2 pour l'aspect interpréter, 4 pour l'aspect intervenir, et 2 pour l'aspect réfléchir. Une échelle à quatre niveaux (débutante [beginning], en 
développement [developing], accomplie [accomplished], exemplaire [exemplary]) permet de qualifier et de quantifier le degré de développement associé aux indicateurs de performance du jugement clinique. Sur le plan quantitatif, pour 11 indicateurs et quatre niveaux, un résultat faible correspond à 11 et un résultat élevé à 44 sur un total de 44 points.

\section{Étape 2 : Traduction renversée}

La version originale en langue anglaise du LCJR a été soumise à un processus de traduction renversée impliquant deux traductrices indépendantes. La version originale a été remise à des traductrices professionnelles bilingues se spécialisant dans la traduction en langue française. Chacune avait un bagage culturel différent, une étant basée au Québec et l'autre au NouveauBrunswick. La première avait l'expérience de traductions en sciences infirmières alors que la deuxième avait fait des traductions d'instruments en pédagogie. Les traductrices n'ont pas échangé durant le processus de traduction. Ces versions ont ensuite été révisées par deux des chercheuses pour en produire une seule. Une chercheuse était une professionnelle de la santé, familière avec la pratique et l'enseignement en sciences infirmières, alors que l'autre était une professionnelle en éducation. Les modifications apportées touchaient à la grammaire (p. ex. utilisation du singulier ou du pluriel) ou certains termes spécifiques (p. ex. utilisation du terme client, patient ou personne). Cette version traduite et révisée en langue française a ensuite été transmise à une troisième traductrice qui l'a traduite à nouveau en langue anglaise. Cette traductrice se spécialisait dans la traduction du français à l'anglais. Elle a eu la consigne de ne pas se référer à la grille originale. Ce processus a résulté en la production de la version préliminaire initiale de la grille.

\section{Étape 3 : Révision de la traduction par un comité}

Un comité d'experts, composé d'une des traductrices, de cinq des membres de l'équipe du projet et de l'auteure de l'instrument, a ensuite délibéré et validé l'équivalence de la version préliminaire en langue française avec l'originale. La participation de l'auteure à la révision de la traduction de l'instrument, bien que n'étant pas toujours réalisable, est préconisée par Hébert et al. (1994). Cela a pour but de maximiser le respect de la signification et de l'intention à l'origine des termes. La participation des traductrices permet de défendre certains choix linguistiques. Toutefois, des contraintes budgétaires ont limité le nombre et le choix (c.-à-d. proximité géographique) à une traductrice invitée. Les ambiguïtés et les divergences relevées à l'étape précédente étaient discutées et résolues par consensus. Une seule rencontre de ce comité a eu lieu. Par la suite, les membres responsables de l'équipe communiquaient au besoin avec l'auteure de l'instrument. Cette révision par comité a permis d'apporter des corrections à la traduction et mené à la version préliminaire du LCJR-Version française (LCJR-VF).

\section{Étape 4 : Prétest}

L'étape suivante du processus a consisté à tester la version préliminaire du LCJR-VF auprès d'un échantillon d'utilisatrices potentielles, soit des enseignantes ou professeures en sciences infirmières ayant une expérience pédagogique de la simulation. La taille d'échantillon recommandée pour un prétest est de 10 à 20 participantes (Hébert et al., 1994). Des membres de l'équipe du projet qui travaillent en simulation ont identifié des participantes potentielles dans leurs réseaux. Ces réseaux couvraient différentes parties de la francophonie. Le recrutement de participantes et la collecte de données pour le prétest se sont effectués entre janvier et mai 2018. Un courriel d'invitation à participer à ce projet a été envoyé, suivi de trois rappels à deux semaines d'intervalle entre chaque envoi. Des données sociodémographiques ont été recueillies, ainsi que le nombre d'années d'expérience en enseignement et le nombre d'années d'expérience en simulation. 
Chaque participante a reçu la version préliminaire en langue française de l'instrument pour l'examiner, sans se référer à l'original. Sa tâche consistait à lire et analyser attentivement le vocabulaire et la grammaire des éléments constituant la grille afin de juger de sa compréhensibilité, particulièrement en lien avec son contexte d'enseignement-apprentissage et de pratique. Une entrevue semi-dirigée individuelle (téléphonique ou en visioconférence) avec un membre de l'équipe de recherche a permis aux participantes de partager leurs impressions générales ainsi que des commentaires spécifiques (p. ex. mots ou expressions difficiles ou inhabituels pour la discipline) et des suggestions pour en améliorer la clarté. Un guide semi-structuré a orienté la discussion, qui était enregistrée sur bande audio, suite à l'obtention du consentement écrit et confirmé oralement par la participante. Le guide d'entrevue était composé de quatre questions portant 1- sur l'impression générale de la grille, 2- sur les mots ou expressions difficiles à comprendre, 3- sur les mots ou expressions inhabituels pour la discipline ou l'expérience en enseignement et 4- sur les suggestions pour clarifier et améliorer la compréhension. Certains mots contestés au moment de la révision des traductions (p. ex. caring, profil clinique, personne au lieu de patient) ont aussi été discutés. Les commentaires répétés relatifs à l'ambiguïté d'un énoncé et les suggestions ont été discutés par l'équipe de recherche et résolus par consensus. Cela a permis d'apporter des corrections supplémentaires à la version traduite du LCJR-VF et de produire une version expérimentale pour la partie suivante, soit l'étape de l'étude de fidélité test-retest.

\section{Étape 5 : Étude de fidélité test-retest}

La dernière étape servait à déterminer la stabilité temporelle (c.-à-d. que l'utilisation de la grille soit la même dans le temps) de la version expérimentale du LCJR-VF au moyen d'une étude de fidélité test-retest.

\section{Échantillon}

La population cible pour cette étude était l'ensemble des enseignantes exerçant en milieu collégial et des professeures en milieu universitaire dans des programmes de formation initiale en sciences infirmières qui utilisent la simulation dans leurs enseignements. Un échantillon par convenance a été constitué (Burns et Grove, 2020; Fortin et Gagnon, 2016). Il s'agit d'un échantillon de type non probabiliste dont le choix des participantes a reposé sur les critères d'inclusion suivants: 1) enseigne dans un domaine de la santé, spécifiquement sciences infirmières; 2) œuvre en tant que « facilitatrice de simulation », 3) participe à l'étude sur une base volontaire, 4) lit et écrit en langue française et 5) est disponible pour les deux étapes de collecte de données à deux semaines d'intervalle. Aucun critère d'exclusion n'a été employé dans le cadre de cette étude. Pour une étude de fidélité test-retest, un échantillon composé de 40 participantes était recommandé afin d'atteindre « un coefficient de corrélation interclasse supérieur à 0,80 avec une probabilité d'erreur de type I de 5 pour cent dans le cadre de deux évaluations [...] Pour ce type d'analyse, la taille de l'échantillon est indépendante du nombre d'items de l'instrument » (Hébert et al., 1994).

\section{Recrutement et collecte de données}

Le recrutement de participantes et la collecte de données pour le test-retest ont été effectués simultanément au Nouveau-Brunswick, au Québec, en Ontario ainsi qu'en Belgique, en France et en Suisse, entre juin 2018 et janvier 2019. Les participantes étaient recrutées parmi les enseignantes et les professeures rattachées aux laboratoires de simulation des établissements d'enseignement supérieur des membres de l'équipe du projet. Deux autres stratégies de recrutement consistaient à inviter les participantes à recommander une collègue ainsi qu'à utiliser 
des communautés de pratique en simulation et nos réseaux sociaux professionnels.

La collecte de données se divisait en deux temps : la formation préalable et le test-retest lui-même. Toutes les participantes à l'étude de fidélité ont été préalablement formées à l'utilisation de l'instrument de mesure afin de standardiser et ainsi minimiser le risque d'introduire un biais d'évaluation (Scriven, 2011). La formation des évaluatrices s'est appuyée sur la méthodologie décrite par Adamson et al. (2012). D'une part, il s'agit de permettre à un grand nombre d'évaluatrices d'observer les mêmes étudiantes prodiguant des soins dans une même situation clinique. Une vidéo modèle montrant des étudiantes prodiguant des soins dans le cadre d'une simulation a été produite pour atteindre ce but. Cette vidéo portait sur le même scénario que celui utilisé pour le test-retest et représentait une équipe de deux étudiantes de $3^{\mathrm{e}}$ année d'un programme de formation initiale. D'autre part, il s'agissait d'offrir une formation aux évaluatrices afin de favoriser le développement d'un cadre de référence commun et de standardiser le plus possible son interprétation. À cette fin, chaque évaluatrice recrutée recevait de l'information écrite, présentée en annexe de cet article, expliquant les composantes de la version expérimentale de la grille et les recommandations d'utilisation ainsi qu'un lien vers la vidéo modèle. Les participantes pouvaient aussi se référer à une grille complétée par trois membres de l'équipe du projet, elles aussi professeures en sciences infirmières et facilitatrices en simulation, pour évaluer la performance de la même équipe.

\section{Instrument}

Chacune des participantes recrutées a été invitée à remplir la version expérimentale du LCJR-VF à deux reprises, chaque fois à la suite du visionnement de trois vidéos présentant les trois temps de la simulation : breffage, simulation et débreffage. Ces vidéos numérisées étaient accessibles en ligne à l'aide d'un code d'accès. Chaque vidéo présentait une équipe de deux étudiantes infirmières d'un programme initial en sciences infirmières d'un de nos établissements. Chaque équipe représentait trois moments différents du parcours de formation $\left(3^{\mathrm{e}}\right.$ année, $4^{\mathrm{e}}$ année, nouvellement diplômée). Un scénario original, ayant fait l'objet d'une validation auprès d'un groupe de discussion de personnes issues des milieux de la santé et de l'éducation, a été choisi pour la simulation (Fournier, 2016). Ce scénario et ses objectifs d'apprentissage abordaient des savoirs précédemment acquis par les étudiantes dans le cadre de leur formation.

Les deux évaluations ont été séparées par un intervalle d'une durée de deux semaines à un mois. Cet intervalle représente le meilleur compromis entre un intervalle court susceptible d'entraîner un biais d'apprentissage ou de mémoire et un intervalle long permettant l'évolution du phénomène mesuré (Hébert et al., 1994; Streiner et al., 2014).

\section{Analyse}

Les données ont été analysées au moyen du logiciel JMP 9.0.0 de SAS Institute Inc. Outre les statistiques descriptives de base, le coefficient $\alpha$ de Cronbach a permis d'estimer la cohérence interne de l'instrument de mesure. Un coefficient $\alpha$ de 0,80 constitue un standard approprié (Adamson et Prion, 2013; Nunnally et Bernstein, 1994). Les coefficients de corrélation de Pearson et de rang (rho de Spearman) ont été calculés pour estimer la fidélité de 1'outil, et les coefficients de corrélation intraclasse avec modèle unidirectionnel d'effets aléatoires ont été calculés pour le test-retest. Le niveau de confiance pour toutes les analyses statistiques est de $95 \%$ et plus ( $p \leq$ $0,05)$. 


\section{Considérations éthiques}

Ce projet a reçu l'approbation des comités d'éthique de la recherche des établissements de chaque membre de l'équipe de recherche (certificats $n^{\circ} 1718-033, n^{\circ} \mathrm{H}-02-18-333$ et $n^{\circ} 14-\mathrm{A} 17$ ). En Suisse, l'approbation éthique n'était pas exigée au sens de la Loi fédérale relative à la recherche sur l'être humain (Gouvernement suisse, 2011). Le projet a aussi reçu l'approbation d'autres établissements ciblés pour le recrutement de participantes. Un formulaire de consentement a été complété par chaque participante avant sa participation au projet.

\section{Résultats}

Les résultats sont présentés en trois sections, soit les étapes de la révision de la traduction, du prétest et de l'étude de fidélité test-retest. Un profil sociodémographique des participantes au prétest et au test-retest est présenté au début de ces sections.

\section{Étape 3 : Révision de la traduction par le comité d'experts}

Le comité d'experts a révisé la grille traduite en s'attardant aux termes qui avaient été traduits différemment par les traductrices ou aux termes qui ne semblaient pas être équivalents à l'intention originale ou représentatifs de la pratique infirmière. Notons en particulier les termes suivants: focused observation, expected patterns, information seeking, patterns in data, intervention, being skillful, prompting et commitment. Les problèmes de traduction ou d'interprétation qui se posaient ont été résolus par consensus. Les résultats des délibérations du comité sont présentés au Tableau 1. Cette révision a permis l'élaboration de la version préliminaire en langue française, soit le LCJR-VF.

\section{Tableau 1}

Termes difficiles à traduire

\begin{tabular}{|c|c|c|}
\hline $\begin{array}{c}\text { Termes de la version } \\
\text { originale }\end{array}$ & Termes suggérés par les traductrices & $\begin{array}{l}\text { Terme choisi par le comité pour } \\
\text { la version préliminaire }\end{array}$ \\
\hline focused observation & observation ciblée & évaluation ciblée \\
\hline expected patterns & $\begin{array}{l}\text { structures prévues, modèles, schémas, } \\
\text { prévisions, écart par rapport aux } \\
\text { prévisions, }\end{array}$ & patterns anticipés \\
\hline information seeking & $\begin{array}{l}\text { recherche de renseignement, recherche } \\
\text { d'information }\end{array}$ & recueillir de l'information \\
\hline patterns in data & $\begin{array}{l}\text { structure dans les données, modèles } \\
\text { dans les données }\end{array}$ & profil clinique \\
\hline $\begin{array}{l}\text { well-planned } \\
\text { intervention/flexibility }\end{array}$ & $\begin{array}{l}\text { intervention planifiée et flexible, } \\
\text { intervention bien panifiée/souplesse }\end{array}$ & intervention planifiée, flexibilité \\
\hline being skillful & $\begin{array}{l}\text { être habile, compétences, maîtrise des } \\
\text { compétences }\end{array}$ & faire preuve de compétence \\
\hline prompting & $\begin{array}{l}\text { incitation, demandé, encouragement, } \\
\text { indices }\end{array}$ & indices \\
\hline commitment & engagement, volonté de s'améliorer & volonté de s'améliorer \\
\hline
\end{tabular}




\section{Étape 4 : Prétest}

Au total, 16 personnes ont participé au prétest. Toutes sont enseignantes ou professeures en sciences infirmières. Toutes sauf deux occupent un poste en milieu universitaire, et toutes sauf une s'identifient au sexe féminin. Dix des participantes proviennent du Canada, trois du Québec, une de l'Ontario et une du Manitoba, et cinq du Nouveau-Brunswick. La Suisse, la France et la Belgique comptent respectivement deux participantes. Le nombre d'années d'expérience en enseignement et en simulation des participantes est présenté à la Figure 1. La majorité des participantes ont entre trois et cinq ans d'expérience en simulation. En ce qui a trait au nombre d'années d'expérience en enseignement, la plupart des participantes ont plus de dix années de pratique.

\section{Figure 1}

Nombre d'années d'expérience des participantes au prétest $(n=16)$

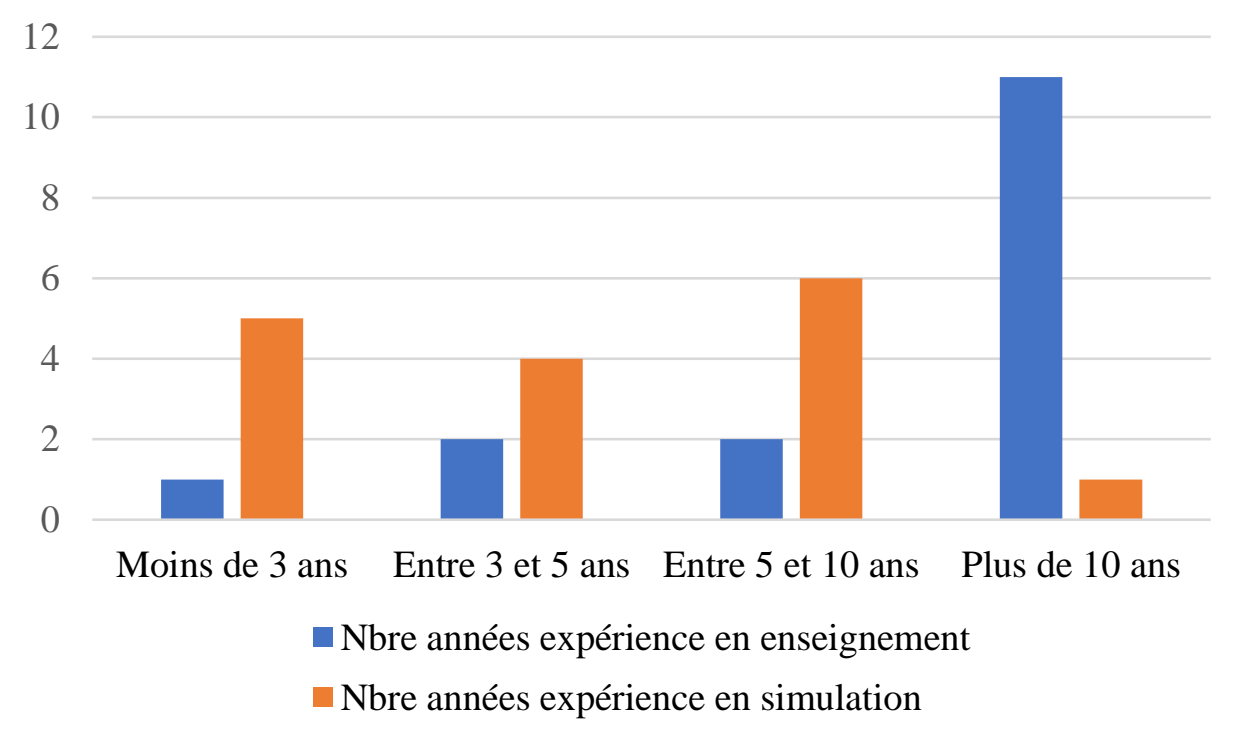

Les entrevues du prétest ont généré quelques suggestions des participantes. Parmi les plus fréquentes et retenues par l'équipe, notons le choix du verbe intervenir plutôt que réagir pour l'aspect responding, l'utilisation de verbes pour nommer chacun des indicateurs, l'utilisation d'autres termes dont «proches » plutôt que «famille » et «état de la personne » plutôt que « diagnostic ». En ce qui a trait à la traduction des termes jugés difficiles à la suite de l'étape de la traduction renversée et de la révision par comité, les participantes avaient différentes suggestions.

Le Tableau 2 en présente quelques exemples ainsi que le terme retenu par l'équipe du projet à la suite de discussions. 


\section{Tableau 2}

Évolution des termes à la suite du prétest

\begin{tabular}{|c|c|c|c|}
\hline $\begin{array}{c}\text { Terme de la version } \\
\text { originale }\end{array}$ & $\begin{array}{c}\text { Terme de la version } \\
\text { préliminaire }\end{array}$ & $\begin{array}{l}\text { Exemples de termes } \\
\text { suggérés par les } \\
\text { participantes au prétest }\end{array}$ & $\begin{array}{l}\text { Terme retenu pour la } \\
\text { version expérimentale }\end{array}$ \\
\hline focused observation & évaluation ciblée & $\begin{array}{l}\text { évaluer de façon ciblée, } \\
\text { exécution de } \\
\text { l'évaluation ciblée }\end{array}$ & évaluer de façon ciblée \\
\hline expected patterns & patterns anticipés & $\begin{array}{l}\text { patterns attendus, } \\
\text { situations typiques, } \\
\text { réactions, réponses, } \\
\text { modèles, schémas, } \\
\text { tendances, patterns } \\
\text { communs }\end{array}$ & profil attendu \\
\hline patterns in data & profil clinique & $\begin{array}{l}\text { état de santé, données } \\
\text { connues, profil de santé }\end{array}$ & profil clinique \\
\hline $\begin{array}{l}\text { well-planned } \\
\text { intervention/flexibility }\end{array}$ & $\begin{array}{l}\text { intervention planifiée, } \\
\text { flexibilité }\end{array}$ & $\begin{array}{l}\text { élaborer un plan de soin } \\
\text { flexible, intervention } \\
\text { planifiée et flexible }\end{array}$ & $\begin{array}{l}\text { intervenir de façon } \\
\text { planifiée et avec } \\
\text { flexibilité }\end{array}$ \\
\hline being skillful & $\begin{array}{l}\text { faire preuve de } \\
\text { compétence }\end{array}$ & $\begin{array}{l}\text { habiletés, habiletés } \\
\text { techniques, compétence, } \\
\text { savoirs }\end{array}$ & $\begin{array}{l}\text { faire preuve de } \\
\text { compétence }\end{array}$ \\
\hline prompting & indices & $\begin{array}{l}\text { besoin d'aide ou } \\
\text { d'assistance, peu de } \\
\text { consultation, peu } \\
\text { d'encouragements }\end{array}$ & $\begin{array}{l}\text { sollicité et } \\
\text { encouragement }\end{array}$ \\
\hline commitment & volonté de s'améliorer & $\begin{array}{l}\text { autonomie, regard } \\
\text { critique, amélioration de } \\
\text { soi, volonté de } \\
\text { progresser }\end{array}$ & $\begin{array}{l}\text { démontrer une volonté } \\
\text { de s'améliorer }\end{array}$ \\
\hline
\end{tabular}

\section{Étape 5 : Étude de fidélité test-retest}

Au total, 35 enseignantes et professeures ont participé à l'étude de fidélité test-retest. Moins de la moitié des participantes a rempli en entier ou partiellement le questionnaire sociodémographique, et ce, malgré les rappels effectués. Une hypothèse expliquant cette situation est que leur participation exigeait un investissement considérable en termes de temps. Bien que court, il est possible que le questionnaire sociodémographique ait été mis de côté en faveur de la demande principale. Des 18 participantes ayant répondu, 14 s'identifient au sexe féminin et une au sexe masculin. Neuf participantes pratiquent au Québec, sept en Suisse, une en Ontario, et une au Nouveau-Brunswick. Onze travaillent en milieu universitaire et sept en milieu collégial. Dix des participantes ont cinq années ou plus d'expérience en enseignement et onze entre trois et dix années d'expérience en simulation (Figure 2). 


\section{Figure 2}

Nombre d'années d'expérience des participantes au test-retest $(n=18)$

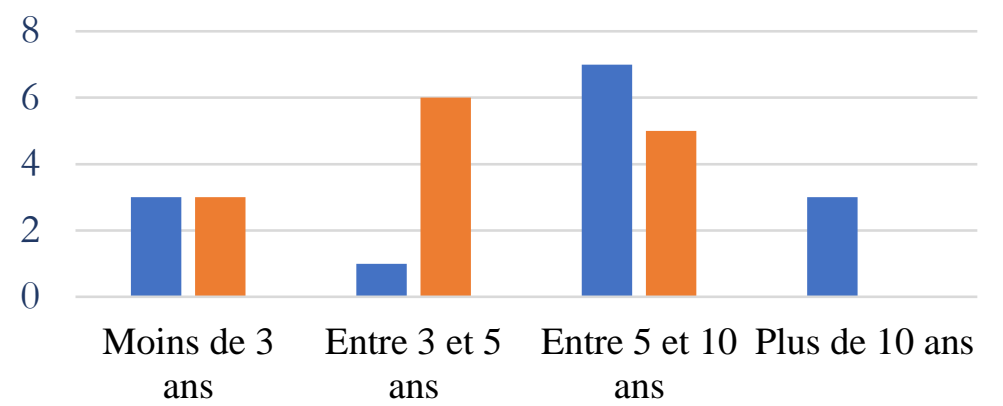

- Nbre années expérience enseignement

Nbre années expérience simulation

La cohérence interne a été évaluée à l'aide du coefficient $\alpha$ de Cronbach. Les coefficients obtenus vont de 0,84 à 0,96 pour les trois vidéos (Tableau 3). Ces résultats font valoir le caractère homogène du contenu de la grille. La stabilité temporelle a été évaluée par un test-retest; le coefficient de corrélation intraclasse avec modèle unidirectionnel d'effets aléatoires a été calculé et va de 0,81 à 0,94 (Tableau 3). Les corrélations test-retest obtenues pour chacune des trois vidéos étaient toutes statistiquement significatives $(\mathrm{p}=<.001)$ confirmant la stabilité temporelle de la grille.

\section{Tableau 3}

Coefficients de corrélation test-retest de trois vidéos

Fiabilité $(\mathrm{n}=33)$

\begin{tabular}{llll}
\hline & $\begin{array}{l}\text { T1 } \\
\text { Cronbach's } \alpha\end{array}$ & $\begin{array}{l}\text { T2 } \\
\text { Cronbach's } \alpha\end{array}$ & $\begin{array}{l}\text { T1-T2 } \\
\text { Intraclass Correlation Coefficient }\end{array}$ \\
\hline Vidéo 1 & 0,93 & 0,93 & $0,87^{*}$ \\
Vidéo 2 & 0,84 & 0,89 & $0,81^{*}$ \\
Vidéo 3 & 0,96 & 0,95 & $0,94^{*}$ \\
\hline Note $* * p=\leq 001$ & &
\end{tabular}

\section{Discussion}

Cet article décrit le processus suivi pour traduire et adapter transculturellement en français un instrument de mesure du jugement clinique, le LCJR. Le modèle de Tanner décrit le jugement clinique d'infirmières d'expérience (Lavoie et al., 2021). Bien qu'il soit utile pour guider et accompagner les étudiantes dans le développement de leur compétence, il ne fournit pas 
d'indication au sujet de la trajectoire du développement. La grille élaborée par Lasater (Cato et al., 2009; Lasater, 2007a) définit cette trajectoire et décrit en termes concrets les quatre aspects du jugement clinique selon le modèle de Tanner (noticing, interpreting, responding, reflecting) facilitant ainsi la compréhension des étudiantes de ce qui est attendu d'elles en termes de performance. La grille permet aussi de concrétiser ce qu'est la réflexion après l'action du modèle de Tanner. Peu importe la situation de soins, la grille décontextualisée de Lasater s'appuie sur des éléments communs du jugement clinique propres au rôle professionnel.

Les résultats du prétest et de l'étude de fidélité test-retest indiquent que cet outil pourrait être utile dans différents contextes francophones de formation infirmière. Les coefficients alpha de Cronbach étaient tous au-dessus de 0,80 , ce qui est un seuil acceptable pour des outils déjà validés et avec une fidélité bien établie comme l'outil LCJR (Adamson et Prion, 2013; Hébert et al., 1994; Fortin et Gagnon, 2016). Román-Cereto et al., (2018) ont traduit le LCJR en langue espagnole et ont obtenu un coefficient alpha de Cronbach de 0,93. De plus, les coefficients de corrélation intraclasse du test-retest pour les trois vidéos étaient tous plus haut que 0,82 . Selon Koo et Li (2016), des coefficients de corrélation intraclasse entre 0,72 et 0,90 sont considérés très bons. Le résultat obtenu est aussi similaire aux résultats d'autres études portant sur la traduction de la grille. Les auteurs de la traduction en langue espagnole (Román-Cereto et al., 2018), néerlandaise (Vreugdenhil et Spek, 2018) et suédoise (Kristiansen et al., 2015) rapportent respectivement des coefficients de corrélation intraclasse de 0,96,0,72, et 0,86. Malgré les subtilités de la langue française et les différences culturelles de nos participantes, les résultats obtenus inspirent confiance quant à la qualité de l'instrument pour apprécier le développement du jugement clinique.

Lasater suggère plusieurs utilisations pour sa grille (Lasater, 2011; Lasater et Nielsen, 2009). C'est avant tout un outil de communication entre professeures et étudiantes afin d'aider les étudiantes à identifier un objectif et établir un plan pour le développement de leur jugement clinique. La grille permet de définir clairement et de façon concrète les attentes et peut être utilisée pour l'évaluation de la performance des étudiantes. Lasater explique que la grille permet de se situer sur la trajectoire du développement du jugement clinique. Finalement, la grille peut servir de guide au débreffage, à la rétroaction et à la réflexion (Al Sabei et Lasater, 2016), incluant l'établissement d'un plan d'apprentissage. Outre son utilisation au moment de la formation initiale, la grille peut s'avérer être bénéfique pour le développement professionnel d'infirmières nouvellement diplômées (Lasater et al., 2015). La grille pourrait aussi être utile en recherche pour des études portant sur l'efficacité d'activités de simulation. La grille a été adaptée de différentes façons. Par exemple, Vreugdenhil et Spek (2018) expliquent leur choix de renverser la présentation des niveaux de développement en allant de « débutante » à « exemplaire » de gauche à droite. Des groupes d'utilisatrices ont aussi suggéré à l'auteure de la grille l'adaptation des dimensions de la grille à des situations cliniques spécifiques et l'intégration d'éléments reliés à la sécurité du patient.

Une limite importante du projet réside dans le nombre de participantes recrutées pour le test-retest. Il est sous la barre des 40 participantes requises pour la puissance de l'étude. De plus, considérant les nombreuses régions de la francophonie, un nombre encore plus élevé de participantes provenant d'autres pays ou régions de la francophonie (Moyen-Orient, Afrique) serait sans nul doute désirable. Une autre limite de l'étude est au niveau du processus de traduction et du choix des traductrices. Sousa et Rojjanasrirat (2011) recommandent le recours à des traductrices ayant une connaissance approfondie de la culture source et de la culture cible. Évidemment, les deux traductrices étaient parfaitement bilingues, mais une seule pouvait revendiquer une connaissance approfondie des deux cultures. Ces mêmes auteurs recommandent 
le recours à plusieurs traductrices à différentes étapes du processus dont la révision de la traduction et la traduction renversée. Toutefois, comme ils le notent, cela exige des ressources additionnelles auxquelles nous n'avions pas accès. Par ailleurs, Sousa et Rojjanasricat suggèrent que l'étape de révision des traductions soit réalisée par une autre traductrice. Dans le cadre de cette étude, la révision des traductions a été faite par deux des membres de l'équipe et une seule traductrice a fait la traduction renversée. En termes de force de l'étude, il y a la participation d'un éventail de participantes francophones à toutes les étapes du processus, tant les membres de l'équipe du projet que les participantes au prétest et au test-retest. Ces personnes provenaient de trois provinces canadiennes ainsi que de pays d'Europe. La participation de l'auteure du LCJR à partir de l'étape de la révision par comité de l'instrument traduit jusqu'à la discussion des résultats est aussi une force. Elle témoignait de la signification, de l'intention et de la nuance des termes utilisés. La participation d'une des traductrices à la révision par comité a aussi permis la justification de choix linguistiques.

\section{Conclusion}

La diversité et la complexité des situations cliniques demandent aux nouvelles infirmières en pratique professionnelle des compétences, dont le jugement clinique, largement développées (Bashford et al., 2012; Deschênes et al., 2016; Lasater et al., 2015; Monagle et al., 2018). Ce développement se réalise notamment par une exposition soutenue aux situations cliniques où l'étudiante est en action puis en réflexion sur l'action. Par conséquent, apprécier le développement du jugement clinique et faciliter ce dernier pendant leur formation est essentiel pour les étudiantes ainsi que les enseignantes et professeures en sciences infirmières. Cet article a décrit la production d'un instrument, le LCJR- version française (LCJR-VF), fondé sur les résultats de la traduction et de la validation dans différentes régions de la francophonie d'un instrument existant, le LCJR, de la langue anglaise à la langue française. À notre connaissance, le LCJR-VF est le premier instrument en langue française décrivant un parcours de développement du jugement clinique, répondant ainsi au besoin d'apprentissage de cette compétence. Il sera intéressant d'explorer et documenter ses utilisations à différents moments du parcours des infirmières (p. ex. formation initiale, orientation et intégration en pratique) ou comme outil d'auto-évaluation en comparaison à son utilisation comme outil de rétroaction. Des adaptations à la terminologie seront peut-être requises pour son utilisation par des étudiantes, enseignantes et professeures d'autres régions de la francophonie. Considérant que beaucoup de simulations se déroulent de plus en plus en contexte interprofessionnel dans la formation initiale des programmes de santé, il serait opportun d'explorer l'adaptation et le transfert de la grille pour d'autres cultures disciplinaires, telles que la médecine et l'inhalothérapie. 


\section{Références}

Adamson, K. A., Gubrud, P., Suderas, S., et Lasater, K. (2012). Assessing the reliability, validity, and use of the Lasater Clinical Judgment Rubric: Three approaches. Journal of Nursing Education, 51(2), 66-73. http://doi.org/10.3928/01484834-20111130-03

Adamson, K. A., Kardong-Edgren, S., et Willhaus, J. (2013). An updated review of published simulation evaluation instruments. Clinical Simulation in Nursing, 9, e393-e400. https://doi.org/10.1016/j.ecns.2012.09.004

Adamson, K., et Prion, S. (2013). Reliability: Measuring internal consistency using Cronbach's alpha. Clinical Simulation in Nursing, 9(5), e179-e180. https://doi.org/10.1016/j.ecns.2012.12.001

Al Sabei, S. D., et Lasater, K. (2016). Simulation debriefing for clinical judgment development: A concept analysis. Nurse Education Today, 45, 42-47. https://doi.org/10.1016/j.nedt.2016.06.008

Arter, J. A., et Chappuis, J. (2006). Creating \& recognizing quality rubrics. Pearson

Bashford, C., Shaffer, B., et Young, C. (2012). Assessment of clinical judgment in nursing orientation: Time well invested. Journal for Nurse in Staff Development, 28, 62-65. https://pubmed.ncbi.nlm.nih.gov/22449878/

Burns, N., et Grove, S. (2020). The Practice of Nursing Research: Appraisal, Synthesis, and Generation of Evidence (9e éd.). Elsevier.

Cato, M. L., Lasater, K., et Peeples, A. I. (2009). Nursing students' self-assessment of their simulation experiences. Nurse Education Perspectives, 30(2), 105-108.

Cloutier, J., Lafrance, J., Michallet, B., Marcoux, L., et Cloutier, F. (2015). French translation and validation of the Readiness for Interprofessional Learning Scale (RIPLS) in a Canadian undergraduate healthcare student context. Journal of Interprofessional Care, 29(2), 150-155. https://doi.org/10.3109/13561820.2014.942837

Côté, C., Gagnon, C., et Payette, H. (2018). Adaptation transculturelle en français du Edinburgh Feeding Evaluation in Dementia (EdFED) Scale : Un questionnaire pour évaluer les difficultés à s'alimenter de personnes âgées présentant des troubles cognitifs en centre d'hébergement. Canadian Journal on Aging, 37(4), 474-481. https://doi.org/10.1017/S0714980818000351

Davis, A. H., et Kimble, L. P. (2011). Human patient simulation evaluation rubrics for nursing education: Mesuring the essentials of baccalaureate education for professional nursing practice. Journal of Nursing Education, 50, 605-611. https://doi.org/10.3928/0148483420110715-01

Deschênes, M.-F., Fournier, V., et St-Julien, A. (2016). Le développement du jugement en situation authentique. Pédagogie Collégiale, 30(1), 14-22.

Fortin, M., et Gagnon, J. (2016). Fondements et étapes du processus de recherche : méthodes quantitatives et qualitatives (3e éd.). Chenelière.

Fournier, V. (2016). Vomissements postopératoires, chirurgie abdominale (iléostomie temporaire). Éducation Montréal. 
Gouvernement Suisse. (2011). Loi fédérale relative à la recherche sur l'être humain.

Confédération suisse. https://www.admin.ch/opc/fr/classifiedcompilation/20061313/index.html

Haccoun, R. (1987). Une nouvelle technique de vérification de l'équivalence de mesures psychologiques traduites. Revue québécois de psychologie, 8(3), 30-39.

Hébert, R., Bravo, G., et Voyer, L. (1994). La traduction d'instruments de mesure pour la recherche gérontologique en langue française : Critères métrologiques et inventaire. Canadian Journal on Aging, 13(3), 392-405. https://doi.org/10.1017/S0714980800006206

Huba, M. E., et Freed, J. E. (2000). Learner-centered assessment on college campuses: shifting the focus from teaching to learning. Allyn \& Bacon.

Kardong-Edgren, S., Adamson, K. A., et Fitzgerald, C. (2010). A review of currently published evaluation instruments for human patient simulation. Clinical Simulation in Nursing, 6(1), e25-e35. https:/doi.org/10.1016/j.ecns.2009.08.004

Kavanagh, J., et Szweda, C. (2017). A crisis in competency: The strategic and ethical imperative to assessing new graduate nurses' clinical reasoning. Nursing Education Perspectives, 38(2), 57-62. https://doi.org/10.1097/01.NEP.0000000000000112

Koo, T. K., et Li, M. Y. (2016). A guideline of selecting and reporting intraclass correlation coefficients for reliability research. Journal of Chiropractic Medicine, 15(2), 155-163. https://doi.org/10.1016/j.jcm.2016.02.012

Kristiansen, L., Häggström, M., Hallin, K., Andersson, I., et Bäckström, B. (2015). Svensk översättning, kvalitativ relevansvärdering och kvantitativ reliabilitetstestning av Lasater Clinical Judgment Rubric: Swedish translation, qualitative relevance evaluation and quantitative reliability test of Lasater Clinical Judgment Rubric. Nordin Journal of Nursing Research, 35(2), 113-122. https://doi.org/10.1177/0107408315578397

Lasater, K. (2007a). Clinical judgment development: Using simulation to create an assessment rubric. Journal of Nursing Education, 46(11), 496-503. https://doi.org/10.3928/01484834-20071101-04

Lasater, K. (2007b). High-fidelity simulation and the development of clinical judgment: Students' experiences. Journal of Nursing Education, 46(6), 269-276. https://doi.org/10.3928/01484834-20070601-06

Lasater, K. (2011). Clinical judgment: The last frontier for evaluation. Nurse Education in Practice, 11, 86-92. https://doi.org/10.1016/j.nepr.2010.11.013

Lasater, K., et Nielsen, A. (2009). Reflective journaling for clinical judgment development and evaluation. Journal of Nursing Education, 48(1), 40-44. https://doi.org/10.3928/01484834-20090101-06

Lasater, K., Nielsen, A. E., Stock, M., et Ostrogorsky, T. L. (2015). Evaluating the clinical judgment of newly hired staff nurses. Journal of Continuing Education in Nursing, 46(12), 563-571. https://doi.org/10.3928/00220124-20151112-09

Lavoie, P., Boyer, L., Pepin, J. I., Goudreau, J., et Fima, O. (2017). Accompagner les infirmières et les étudiantes dans la réflexion sur des situations de soins : Un modèle pour les 
formateurs en soins infirmiers. Quality Advancement in Nursing Education - Avancées en formation infirmière, 3(1), 1-14. https://doi.org/10.17483/2368-6669.1100

Lavoie, P., Deschênes, M.-F., Richard, V., Pepin, J. I., Tanner, C. A., et Lasater, K. (2021). Traduction et adaptation d'un modèle du jugement clinique infirmier pour la recherche et la formation infirmière en contexte francophone. Quality Advancement in Nursing Education - Avancées en formation infirmière, 7(2).

Lavoie, P., Pepin, J., et Cossette, S. (2015). Development of a post-simulation debriefing for intervention to prepare nurses and nursing students to care for deteriorating patients. Nurse Education in Practice, 15, 181-191. https://doi.org/10.1016/j.nepr.2015.01.006

Lesmond, G., McCahan, S., et Beach, D. (2017). Élaboration de rubriques d'analyse pour mesurer les compétences. Conseil ontarien de la qualité de l'enseignement supérieur.

Mariani, B., Cantrell, M. A., Meakim, C., Prieto, P., et Dreifuerst, K. T. (2013). Structured debriefing and students' clinical judgment abilities in simulation. Clinical Simulation in Nursing, 9(5), e147-e155. https://doi.org/10.1016/j.ecns.2011.11.009

Monagle, J., Lasater, K., Stoyles, S., et Dieckmann, N. (2018). New graduate nurse experiences in clinical judgment: What academic and practice educators need to know. Nursing Education Perspectives, 39(4), 201-207. https://doi.org/10.1097/01.NEP.0000000000000336

Nunes, J. G., Lasater, K., Oliveira-Kumakura, A. R., Garbuio, D. C., Braga, F. T., et de Carvalho, E. C. (2016). Adaptation of the Lasater Clinical Judgment Rubric to the Brazilian culture. Journal of Nursing, 10(Suppl. 6), 4828-4836.

Nunnally, J., et Bernstein, I. (1994). Psychometric Theory (3e éd.). McGraw-Hill.

Parker, V., Giles, M., Lantry, G., et McMillan, M. (2014). New graduate nurses' experiences in their first year of practice. Nurse Education Today, 34(1), 150-156. https://doi.org/10.1016/j.nedt.2012.07.003

Román-Cereto, M., García-Mayor, S., Kaknani-Uttumchandani, S., García-Gámez, M., LeónCampos, A., Fernández-Ordóñez, E., Ruiz-García, M. R., Martí-García, C., López-Leiva, I., Lasater, K., et Morales-Asencio, J. M. (2018). Cultural adaptation and validation of the Lasater Clinical Judgment Rubric in nursing students in Spain. Nurse Education Today, 64, 71-78. https://doi.org/10.1016/j.nedt.2018.02.002

Scriven, M. (2011). Evaluation bias and its control. Journal of Multidisciplinary Evaluation, 7(15), 79-98.

Shin, H., Park, C. G., et Shim, K. (2015). The Korean version of the Lasater Clinical Judgment Rubric: A validation study. Nurse Education Today, 35, 68-72. https://doi.org/10.1016/j.nedt.2014.06.009

Sideras, S. (2007). An examination of the construct validity of a clinical judgment evaluation tool in the setting of high-fidelity simulation. Thèse de doctorat non publiée. Oregon Health \& Science University.

Simoneau, I. L., et Paquette, C. (2014). Pédagogie par la simulation clinique haute fidélité dans la formation collégiale en santé. Rapport de recherche PAREA PA2012-015, Cégep de Sherbrooke. 
Simoneau, I. L., Ledoux, I., et Paquette, C. (2012). Efficacité pédagogique de la simulation clinique haute fidélité dans le cadre de la formation collégiale en soins infirmiers. Rapport de recherche PAREA PA2010-004, Cégep de Sherbrooke.

Sousa, V. D., et Rojjanasrirat, W. (2011). Translation, adaptation and cultural validation of intruments or scales for use in cross-cultural health care research: A clear and userfriendly guide. Journal of Evaluation in Clinical Practice, 17, 268-274. https://doi.org/10.1111/j.1365-2753.2010.01434.x

Streiner, D., Norman, G., et Cairney, J. (2014). Health Measurement Scales. A Practical Guide to Their Development and Use (4e éd.). Oxford University Press.

Tanner, C. A. (2006). Thinking like a nurse: A research-based model of clinical judgment in nursing. Journal of Nursing Education, 45(6), 204-211. https://doi.org/10.3928/01484834-20060601-04

Tardif, J. (2006). L'évaluation des compétences : Documenter le parcours de développement. Chenelière Éducation.

Vallerand, R. (1989). Vers une méthodologie de validation transculturelle de questionnaires psychologiques : Implications pour la recherche en langue française. Psychologie canadienne, 30(4), 662-680. https://doi.org/10.1037/h0079856

Vreugdenhil, J., et Spek, B. (2018). Development and validation of Dutch version of Lasater Clinical Judgment Rubric in hospital practice: An instrument design study. Nurse Education Today, 62, 43-51. https://doi.org/10.1016/j.nedt.2017.12.013

Yang, F., Wang, Y., Yang, C., Zhou, M. H., Shu, J., Fu, B., et Hu, H. (2019). Improving clinical judgment by simulation: A randomized trial validation of the Lasater clinical judgment rubric in Chinese. BMC Medical Education, 19(20), 1-6. https://doi.org/10.1186/s12909$\underline{019-1454-9}$ 


\section{Annexe : Guide explicatif d'utilisation et grille LCJR-VF}

\section{Développement du jugement clinique : traduction et adaptation française de la grille du jugement clinique de Lasater ${ }^{\circledR}($ LCJR-VF)}

Équipe du projet : Jacinthe Beauchamp ${ }^{1}$, Michelle Lalonde ${ }^{2}$, Viviane Fournier ${ }^{3}$, Sabrina Mehiz ${ }^{4}$, Marco Pedrotti ${ }^{4}$, Isabelle Michel $^{5}$, Pierre Godbout ${ }^{6}$, Ivan Simoneau ${ }^{7}$

${ }^{1}$ Centre de formation médicale du Nouveau-Brunswick, Moncton, NB

${ }^{2}$ École de sciences infirmières, Université d'Ottawa, ON

${ }^{3}$ Programme de sciences infirmières, Université du Québec en Abitibi-Témiscamingue, QC

${ }^{4}$ Haute École Arc Santé, HES-SO Neuchâtel, Suisse

${ }^{5}$ Programme sciences infirmières, Collège Boréal, Sudbury, ON

${ }^{6}$ École de science infirmière, Université de Moncton, Moncton, NB

${ }^{7}$ Centre de recherche de formation par simulation (CEREFS), Sherbrooke, QC

Remerciements :

- À toutes les participantes et à tous les participants au processus de validation

- Aux étudiantes et aux étudiants qui ont participé à la simulation et qui ont permis la réalisation des capsules vidéo inhérentes à la validation de la grille et à cette formation

- À Pre Kathie Lasater, Ph. D., pour son outil original et pour sa grande disponibilité tout au long du processus de traduction, adaptation et validation

- Aux organismes qui ont rendu ce projet possible grâce à leur financement : le Bureau de recherche du Centre de formation médicale du N.-B., le Réseau-action Formation et recherche de la Société Santé et Mieux-être en français du N.-B et la Haute Ecole Spécialisée de Suisse occidentale HES-SO 


\section{Introduction}

L'apprentissage par simulation est à ce jour une modalité pédagogique incontournable dans les programmes de formation en sciences de la santé nécessitant le développement du jugement clinique. Pourtant, à ce jour, le corps professoral recherche toujours des outils valides en français permettant d'accompagner les étudiantes dans le développement de leurs compétences contextualisées à la diversité des situations professionnelles. Pour combler en partie ce besoin concernant l'évaluation formative du développement du jugement clinique, une équipe canadosuisse multisites a traduit, adapté et validé en langue française le Lasater Clinical Judgment Rubric. Cet outil validé est utilisé aux États-Unis et, depuis sa diffusion originale en 2007, a été traduit et adapté par des équipes de plusieurs pays dont le Brésil, la Chine, la Corée, l'Espagne, les Pays-Bas et la Suède.

\section{Objectifs du guide d'utilisation}

En conformité avec la vision du Pre Lasater, il est important que tous ceux et celles qui utiliseront la grille aient une compréhension commune de ses composantes et de ses recommandations d'utilisation. Nous vous encourageons fortement à lire attentivement ce document. Il vous permettra de vous familiariser, ainsi que vos collègues qui l'utiliseront, avec la grille et ses intentions.

\section{Conception de la grille}

La grille du jugement clinique a été conçue par Pre Kathie Lasater pour apprécier la trajectoire du développement du jugement clinique de l'étudiante en sciences infirmières à travers son cheminement dans son programme de formation. Elle offre aux étudiantes, aux professeures et aux monitrices cliniques un langage commun pour discuter du raisonnement des étudiantes dans le cadre d'activités formatives et réflexives ${ }^{2}$. Jugée fiable et valide dans le cadre d'autres études, et déjà traduite en plusieurs langues, cette grille est basée sur le cadre conceptuel du jugement clinique de Tanner $(2006)^{3}$.

Elle permet de comprendre et de positionner les quatre principaux aspects du jugement clinique :

- Remarquer

- Interpréter

- Intervenir

- Réfléchir

Chaque aspect est détaillée à l'aide de 2 à 4 dimensions du jugement clinique. L'échelle de stades développementaux est à quatre niveaux :

- Débutante

- En développement

- Accomplie

\footnotetext{
${ }^{2}$ Lasater (2007a). Clinical judgment development: Using simulation to create an assessment rubric. Journal of Nursing Education, 46, 11, 496-503. Lasater (2011). Clinical judgment: The last frontier for evaluation. Nurse Education in Practice, 11, 86-92.

3 Tanner, C.A. (2006). Thinking like a nurse: A research-based model of clinical judgment in nursing. Journal of Nursing Education, 45, 6, 204-211.
} 


\section{- Exemplaire}

Une description pour chaque dimension sous chacun des niveaux de développement permet d'identifier le niveau atteint lors de l'observation d'une simulation, incluant les parties du breffage et du débreffage.

La grille est construite selon la forme typique, c'est-à-dire avec les aspects et dimensions à gauche (lignes) et les niveaux de développement en haut (colonnes). Les niveaux vont du plus haut au plus bas, le dernier niveau à gauche (Exemplaire), étant la cible ultime à atteindre.

\section{Utilisation de la grille pour enseigner et apprendre le jugement clinique, en évaluation formative ou en auto-évaluation}

Comme le disent Tanner (2006) et Lasater (Cato et al., 2009; Lasater, 2007a) définit ellesmêmes, l'utilisation de la grille permet d'aider les étudiantes à « apprendre à raisonner comme une infirmière ». La grille clarifie ce qu'on entend par jugement clinique, mais permet aussi aux étudiantes de juger leur niveau de développement et d'identifier des points à améliorer à chacune de ses utilisations.

La grille est construite pour des étudiantes en formation. En début de formation, on s'attend donc à ce qu'elles soient au niveau « débutante » alors qu'en fin de formation, elles devraient être minimalement au niveau « accomplie ». Évidemment, la diversité du corps étudiant signifie qu'à la fin de leur programme, voire avant, certaines étudiantes pourraient atteindre le niveau accomplie ou même exemplaire pour certaines dimensions, par exemple communiquer clairement ou évaluer et s'autoévaluer. Ainsi, la grille peut être utilisée par une professeure pour évaluer de façon formative et constituer une base à l'accompagnement pédagogique nécessaire au développement de cette compétence complexe. La grille peut également être utile au développement des stratégies métacognitives en offrant aux étudiantes un outil de réflexion après l'action.

\section{Appropriation préalable : formation à l'utilisation de la grille}

Nous mettons à votre disposition une vidéo d'environ 45 minutes (veuillez communiquer avec la responsable du projet pour y accéder) pour favoriser votre compréhension de la grille et de son utilisation. En visionnant la vidéo, nous vous recommandons d'utiliser la grille pour apprécier la performance d'une dyade étudiante. Vous pouvez ensuite comparer votre appréciation à celle complétée par un groupe d'experts l'ayant utilisée dans le cadre du projet de traduction et de validation (voir le tableau à la suite de la grille). Trois professeures en sciences infirmières ont constitué ce groupe.

Dans la vidéo, vous pourrez observer deux étudiantes en sciences infirmières et leur professeure lors d'une activité de simulation, en trois temps. L'activité débute avec un breffage, période durant lequel la professeure présente l'activité d'apprentissage et la situation clinique. Elle invite ensuite l'équipe à formuler des hypothèses et planifier des interventions possibles en lien avec ces hypothèses. Une étudiante alors identifiée agit à titre de leader avec la responsabilité ultime des soins au patient, incluant la délégation de tâches à sa coéquipière. Seulement l'étudiante qui agit à titre de leader est évaluée avec la grille.

\section{Utilisation de la grille}

Écoutez attentivement son discours pour des indices de jugement clinique. À l'aide de la grille, procédez à une appréciation initiale de son jugement clinique avant, pendant et lors du débreffage, suite à la simulation. Vous pouvez encercler vos choix sur une version papier de la 
grille ou surligner vos choix dans une version électronique. Comparez ensuite votre évaluation et utilisation de la grille avec celle du groupe d'experts. Vos noterez que pour certaines dimensions, l'appréciation du niveau de développement a été majoritaire mais non unanime, faisant état du jugement de l'instructrice.

Il se peut que vous remarquiez un accent francophone différent du vôtre chez les étudiantes de la vidéo. Vous noterez également que les étudiantes ou la professeure utilisent des expressions ou des façons de faire propres à leur milieu (p. ex. tutoiement du patient). Ces différences reflètent les besoins d'adaptation culturelle et de contextualisation des soins de santé au Canada français ou dans la francophonie internationale.

\section{Droits d'utilisation}

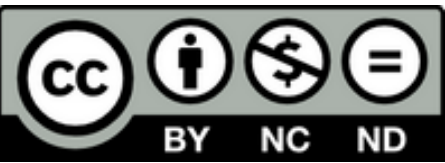

Vous pouvez copier, distribuer et communiquer le matériel par tous moyens et sous tous formats.

Restrictions (3) :

- Vous devez créditer l'œuvre et intégrer un lien vers la licence.

- Vous n'êtes pas autorisé à faire un usage commercial de cette œuvre.

- Dans le cas où vous effectuez un remix, une transformation ou une nouvelle création basée sur du matériel composant l'œuvre originale, vous n'êtes pas autorisé à distribuer ou mettre à disposition l'œuvre modifiée.

Pour toutes autres utilisations ou toutes communications concernant les droits d'utilisation, ou pour tout commentaire à la suite de l'utilisation de la grille, nous vous prions de communiquer avec la responsable du projet : jacinthe.beauchamp@umoncton.ca.

Merci de votre intérêt !

L'équipe du projet 


\section{GRILLE DU JUGEMENT CLINIQUE DE LASATER : LCJR-VF}

Remarquer et interpréter

\begin{tabular}{|c|c|c|c|c|}
\hline $\begin{array}{l}\text { REMARQUER } \\
\text { efficacement nécessite : }\end{array}$ & Exemplaire & Accomplie & En développement & Débutante \\
\hline Évaluer de façon ciblée & $\begin{array}{l}\text { Cible adéquatement son évaluation; } \\
\text { évalue et surveille régulièrement un } \\
\text { large éventail de données } \\
\text { subjectives et objectives afin } \\
\text { d'obtenir toutes les informations } \\
\text { utiles. }\end{array}$ & $\begin{array}{l}\text { Évalue et surveille régulièrement une } \\
\text { variété de données subjectives et } \\
\text { objectives; les informations utiles sont } \\
\text { presque toutes recueillies, mais manque } \\
\text { à l'occasion des signes plus subtils. }\end{array}$ & $\begin{array}{l}\text { Surveille une variété de données } \\
\text { subjectives et objectives, mais est } \\
\text { dépassée par le large éventail de } \\
\text { données; se concentre sur les données } \\
\text { les plus évidentes, manquant plusieurs } \\
\text { informations importantes. }\end{array}$ & $\begin{array}{l}\text { Est déconcertée par la situation clinique } \\
\text { et la quantité ou le type de données; } \\
\text { l'évaluation est désordonnée; manque } \\
\text { des données importantes ou commet des } \\
\text { erreurs d'évaluation. }\end{array}$ \\
\hline $\begin{array}{l}\text { Reconnaître des écarts par } \\
\text { rapport au profil attendu }\end{array}$ & $\begin{array}{l}\text { Reconnaît les écarts subtils par } \\
\text { rapport au profil attendu et s'en sert } \\
\text { pour guider l'évaluation. }\end{array}$ & $\begin{array}{l}\text { Reconnait les écarts les plus évidents par } \\
\text { rapport au profil attendu et s'en sert pour } \\
\text { continuer l'évaluation. }\end{array}$ & $\begin{array}{l}\text { Identifie les écarts évidents par rapport } \\
\text { au profil attendu; manque des } \\
\text { informations importantes; est incertaine } \\
\text { comment continuer l'évaluation. }\end{array}$ & $\begin{array}{l}\text { Se concentre sur une chose à la fois et } \\
\text { manque la plupart des écarts par rapport } \\
\text { au profil attendu; manque des occasions } \\
\text { de bonifier l'évaluation. }\end{array}$ \\
\hline Recueillir de l'information & $\begin{array}{l}\text { Recueille avec assurance des } \\
\text { informations afin de planifier ses } \\
\text { interventions; recueille } \\
\text { judicieusement des données } \\
\text { subjectives utiles en observant et en } \\
\text { interagissant avec la personne et ses } \\
\text { proches. }\end{array}$ & $\begin{array}{l}\text { Recueille activement des informations } \\
\text { subjectives auprès de la personne et de } \\
\text { ses proches afin de l'aider à planifier ses } \\
\text { interventions; ne poursuit pas toujours } \\
\text { des données importantes. }\end{array}$ & $\begin{array}{l}\text { Déploie peu d'efforts pour trouver des } \\
\text { informations supplémentaires auprès de } \\
\text { la personne et de ses proches; souvent, } \\
\text { ne semble pas savoir quelles } \\
\text { informations recueillir ou poursuit des } \\
\text { données non pertinentes. }\end{array}$ & $\begin{array}{l}\text { Est inefficace dans sa recherche } \\
\text { d'informations; se base principalement } \\
\text { sur des données objectives; éprouve de } \\
\text { la difficulté à interagir avec la personne } \\
\text { et ses proches et ne recueille pas des } \\
\text { données subjectives importantes. }\end{array}$ \\
\hline $\begin{array}{l}\text { INTERPRÉTER } \\
\text { efficacement nécessite : }\end{array}$ & Exemplaire & Accomplie & En développement & Débutante \\
\hline Prioriser les données & $\begin{array}{l}\text { Se concentre sur les données les } \\
\text { plus pertinentes et importantes qui } \\
\text { sont utiles pour expliquer l'état de } \\
\text { la personne. }\end{array}$ & $\begin{array}{l}\text { Se concentre principalement sur les } \\
\text { données les plus importantes et } \\
\text { recherche d'autres informations } \\
\text { significatives, mais s'attarde parfois } \\
\text { sur des données moins pertinentes. }\end{array}$ & $\begin{array}{l}\text { Tente de prioriser les données et de se } \\
\text { concentrer sur les plus importantes } \\
\text { d'entre elles, mais s'attarde également } \\
\text { sur les données moins importantes ou } \\
\text { pertinentes. }\end{array}$ & $\begin{array}{l}\text { Éprouve de la difficulté à se } \\
\text { concentrer sur les données et ne } \\
\text { semble pas savoir lesquelles sont } \\
\text { importantes en lien avec l'état de la } \\
\text { personne; tente de composer avec } \\
\text { toutes les données disponibles. }\end{array}$ \\
\hline
\end{tabular}




\begin{tabular}{|c|c|c|c|c|}
\hline $\begin{array}{l}\text { Comprendre } \\
\text { les données }\end{array}$ & $\begin{array}{l}\text { Lorsque confrontée à des données } \\
\text { complexes, contradictoires ou } \\
\text { confondantes, est capable de (1) } \\
\text { noter et comprendre le profil } \\
\text { clinique de la personne, (2) } \\
\text { comparer ce dernier avec des } \\
\text { profils connus (issus des } \\
\text { connaissances en sciences } \\
\text { infirmières, de la recherche, de } \\
\text { l'expérience personnelle et de } \\
\text { l'intuition) et (3) élaborer des } \\
\text { plans d'intervention qui sont } \\
\text { justifiés et réalistes. }\end{array}$ & $\begin{array}{l}\text { Dans la plupart des situations, } \\
\text { interprète le profil clinique de la } \\
\text { personne et le compare aux profils } \\
\text { connus afin d'élaborer un plan } \\
\text { d'intervention justifié; dans les cas } \\
\text { complexes ou rares recherche } \\
\text { l'expertise d'une spécialiste ou d'une } \\
\text { infirmière plus expérimentée. }\end{array}$ & $\begin{array}{l}\text { Dans des situations simples, } \\
\text { communes ou familières, est capable } \\
\text { de comparer le profil clinique de la } \\
\text { personne avec des profils connus et } \\
\text { d'élaborer et de justifier un plan } \\
\text { d'intervention; éprouve de la } \\
\text { difficulté avec des données ou des } \\
\text { situations moindrement difficiles qui } \\
\text { sont au niveau des attentes; requiert } \\
\text { des conseils ou de l'aide. }\end{array}$ & $\begin{array}{l}\text { Dans des situations simples, } \\
\text { communes ou familières, éprouve de } \\
\text { la difficulté à comprendre et } \\
\text { interpréter les données; démontre de la } \\
\text { difficulté à faire la distinction entre les } \\
\text { explications divergentes et les } \\
\text { interventions appropriées et requiert de } \\
\text { l'aide pour comprendre l'état de la } \\
\text { personne et pour élaborer une } \\
\text { intervention. }\end{array}$ \\
\hline
\end{tabular}

\section{Intervenir et réfléchir}

\begin{tabular}{|c|c|c|c|c|}
\hline $\begin{array}{l}\text { INTERVENIR } \\
\text { efficacement nécessite : }\end{array}$ & Exemplaire & Accomplie & En développement & Débutante \\
\hline $\begin{array}{l}\text { Agir calmement et avec } \\
\text { confiance }\end{array}$ & $\begin{array}{l}\text { Assume ses responsabilités; } \\
\text { délègue des tâches à l'équipe; } \\
\text { évalue et rassure la personne et ses } \\
\text { proches. }\end{array}$ & $\begin{array}{l}\text { Exerce généralement son leadership, } \\
\text { fait preuve de confiance en soi et } \\
\text { arrive à gérer la plupart des } \\
\text { situations; peut démontrer du stress } \\
\text { lors de situations difficiles ou } \\
\text { complexes. }\end{array}$ & $\begin{array}{l}\text { Hésite à assumer son rôle de leader; } \\
\text { rassure la personne et ses proches } \\
\text { dans des situations de routine ou } \\
\text { relativement simples, mais devient } \\
\text { facilement stressée et désorganisée. }\end{array}$ & $\begin{array}{l}\text { Sauf dans des situations de routine } \\
\text { ou relativement simples, est } \\
\text { stressée et désorganisée, perd le } \\
\text { contrôle; rend la personne et ses } \\
\text { proches anxieux et moins aptes à } \\
\text { collaborer. }\end{array}$ \\
\hline Communiquer clairement & $\begin{array}{l}\text { Communique efficacement; } \\
\text { explique ses interventions; rassure la } \\
\text { personne et ses proches; dirige et } \\
\text { implique les membres de l'équipe } \\
\text { en leur donnant des explications et } \\
\text { les guidant; valide leur } \\
\text { compréhension. }\end{array}$ & $\begin{array}{l}\text { Communique généralement bien; } \\
\text { fournit des explications à la } \\
\text { personne, donne des directives } \\
\text { claires à l'équipe; pourrait être plus } \\
\text { efficace à établir un rapport. }\end{array}$ & $\begin{array}{l}\text { Démontre des habiletés de base en } \\
\text { communication (p. ex. donner des } \\
\text { directives); les communications avec } \\
\text { la personne, ses proches et les } \\
\text { membres de l'équipe sont à améliorer; } \\
\text { fait preuve de bienveillance plus que } \\
\text { de compétence. }\end{array}$ & $\begin{array}{l}\text { Communique avec difficulté; ses } \\
\text { explications portent à confusion, ses } \\
\text { directives sont peu claires ou } \\
\text { contradictoires; crée de la confusion } \\
\text { et de l'anxiété chez la personne et ses } \\
\text { proches au lieu de les mettre en } \\
\text { confiance. }\end{array}$ \\
\hline $\begin{array}{l}\text { Intervenir de façon } \\
\text { planifiée et avec } \\
\text { flexibilité }\end{array}$ & $\begin{array}{l}\text { Adapte ses interventions à chaque } \\
\text { personne; surveille de près ses progrès } \\
\text { et ajuste l'intervention selon la } \\
\text { réaction de la personne. }\end{array}$ & $\begin{array}{l}\text { Élabore des interventions sur la base } \\
\text { des données pertinentes à la personne; } \\
\text { surveille régulièrement le progrès, } \\
\text { mais n'anticipe pas le besoin d'ajuster } \\
\text { les interventions. }\end{array}$ & $\begin{array}{l}\text { Élabore des interventions sur la base } \\
\text { des données les plus évidentes; } \\
\text { surveille le progrès, mais est incapable } \\
\text { d'ajuster l'intervention selon la } \\
\text { réaction de la personne. }\end{array}$ & $\begin{array}{l}\text { Se concentre sur l'élaboration d'une } \\
\text { seule intervention qui traite d'une } \\
\text { solution possible, mais elle pourrait } \\
\text { être vague, incomplète ou porter à } \\
\text { confusion; un certain suivi pourrait } \\
\text { avoir lieu. }\end{array}$ \\
\hline
\end{tabular}


Quality Advancement in Nursing Education - Avancées en formation infirmière, Vol. 7, Iss. 2 [2021], Art. 5

\begin{tabular}{|c|c|c|c|c|}
\hline Faire preuve de compétence & $\begin{array}{l}\text { Démontre la maîtrise nécessaire } \\
\text { des compétences infirmières. }\end{array}$ & $\begin{array}{l}\text { Utilise la plupart des compétences } \\
\text { infirmières; pourrait améliorer sa } \\
\text { rapidité ou sa précision. }\end{array}$ & $\begin{array}{l}\text { Est hésitante ou inefficace dans } \\
\text { sa démonstration de } \\
\text { compétences infirmières. }\end{array}$ & $\begin{array}{l}\text { Est incapable de choisir ou } \\
\text { d'appliquer les compétences } \\
\text { infirmières }\end{array}$ \\
\hline $\begin{array}{l}\text { RÉFLÉCHIR } \\
\text { efficacement nécessite : }\end{array}$ & Exemplaire & Accomplie & En développement & Débutante \\
\hline Évaluer et s'autoévaluer & $\begin{array}{l}\text { Évalue/analyse de manière } \\
\text { indépendante sa performance, ses } \\
\text { prises de décisions, élabore des } \\
\text { solutions de rechange et évalue } \\
\text { judicieusement ses choix et les } \\
\text { compare aux solutions de rechange. }\end{array}$ & $\begin{array}{l}\text { Évalue et analyse sa performance } \\
\text { avec peu d'encouragement, examine } \\
\text { principalement les décisions ou } \\
\text { événements majeurs; identifie les } \\
\text { décisions clés et envisage des } \\
\text { solutions de rechange. }\end{array}$ & $\begin{array}{l}\text { Même lorsque sollicitée, verbalise } \\
\text { brièvement les évaluations les plus } \\
\text { évidentes; envisage difficilement des } \\
\text { solutions de rechange; justifie ses } \\
\text { décisions de manière défensive. }\end{array}$ & $\begin{array}{l}\text { Même lorsque sollicitée, ses } \\
\text { évaluations sont brèves, sommaires } \\
\text { et ne servent pas à l'amélioration de } \\
\text { sa performance; justifie de manière } \\
\text { défensive ses décisions et ses choix } \\
\text { sans les évaluer. }\end{array}$ \\
\hline $\begin{array}{l}\text { Démontrer une } \\
\text { volonté de } \\
\text { s'améliorer }\end{array}$ & $\begin{array}{l}\text { Démontre un engagement continu à } \\
\text { s'améliorer : réfléchit et évalue de } \\
\text { manière critique ses expériences; } \\
\text { identifie avec justesse ses forces et } \\
\text { ses points à améliorer et élabore des } \\
\text { stratégies pour s'améliorer. }\end{array}$ & $\begin{array}{l}\text { Démontre une volonté d'améliorer } \\
\text { sa performance : réfléchit et évalue } \\
\text { ses expériences; identifie ses forces } \\
\text { et ses points à améliorer; pourrait } \\
\text { être plus systématique dans } \\
\text { l'évaluation de ses points à } \\
\text { améliorer. }\end{array}$ & $\begin{array}{l}\text { Reconnaît qu'il y a place à } \\
\text { l'amélioration et fait certains efforts } \\
\text { pour apprendre de son expérience et } \\
\text { améliorer sa performance, mais se } \\
\text { limite à des conclusions évidentes et } \\
\text { a besoin d'un regard externe. }\end{array}$ & $\begin{array}{l}\text { Ne démontre pas d'intérêt à } \\
\text { améliorer sa performance ou est } \\
\text { incapable de le faire; réfléchit peu; } \\
\text { ne fait aucune autocritique ou est } \\
\text { trop critique (par rapport à son } \\
\text { niveau d'apprentissage); n'identifie } \\
\text { pas ses points à améliorer ou la } \\
\text { nécessité de s'améliorer. }\end{array}$ \\
\hline
\end{tabular}




\section{Appréciation et justification de trois professeures expertes}

(À noter que les commentaires s'appliquent parfois aux actions des deux étudiantes.)

\begin{tabular}{|c|c|c|}
\hline $\begin{array}{l}\text { Aspect et } \\
\text { dimensions }\end{array}$ & $\begin{array}{l}\text { Évaluation } \\
\text { (Accord } \\
\text { interjuge) }\end{array}$ & Justification \\
\hline \multicolumn{3}{|l|}{ REMARQUER } \\
\hline $\begin{array}{l}\text { Évaluer de façon } \\
\text { ciblée }\end{array}$ & $\begin{array}{c}\text { En } \\
\text { développement } \\
(2 / 3)\end{array}$ & $\begin{array}{l}\text { 1- Collecte de données un peu désorganisée et incomplète, par exemple l'évaluation de } \\
\text { la douleur était incomplète en dépit du fait que le client se plaint de façon répétée. } \\
\text { Même si c'est la collègue qui avait cette tâche, il fallait s'assurer qu'elle soit } \\
\text { accomplie. } \\
\text { 2- Les éléments les plus importants de la fonction abdominale sont évalués sauf } \\
\text { percussion et palpation. Quelques éléments de l'évaluation de la douleur sont } \\
\text { manquants (temps, provoquer/pallier). } \\
\text { 3- Simulation : L'évaluation est un peu désorganisée, la mesure des signes vitaux n'était } \\
\text { pas forcément prioritaire ou aurait pu être déléguée. } \\
\text { Débreffage : Bonne évaluation de la situation, toutes les données étaient utiles. }\end{array}$ \\
\hline $\begin{array}{l}\text { Reconnaitre des } \\
\text { écarts par rapport } \\
\text { au profil attendu }\end{array}$ & $\begin{array}{c}\text { En } \\
\text { développement } \\
(2 / 3)\end{array}$ & $\begin{array}{l}\text { 1- Était incertaine quant à la façon de continuer l'évaluation dans le contexte du } \\
\text { vomissement et la présence de l'épouse. A toutefois été capable d'identifier des } \\
\text { écarts pertinents. } \\
\text { 2- Établit le besoin prioritaire nausée, mais pas le besoin de mettre NPO. } \\
\text { 3- Simulation : le manque de connaissances parasite la prise de décision en regard de la } \\
\text { situation. }\end{array}$ \\
\hline $\begin{array}{l}\text { Recueillir de } \\
\text { l'information }\end{array}$ & $\begin{array}{c}\text { En } \\
\text { développement } \\
(2 / 3)\end{array}$ & $\begin{array}{l}\text { 1- La présence de l'épouse semble avoir déboussolé l'étudiante. Peu de questions ont } \\
\text { été posées au client et à son épouse sur les symptômes, etc. } \\
\text { 2- Aurait pu valider l'info voulant que la femme se dise préoccupée de faire un pas en } \\
\text { arrière. } \\
\text { 3- Simulation : inconfortable avec la présence de la famille, n'ose pas intervenir par } \\
\text { rapport au jus d'orange et au café. Pas sûre que ça ne soit pas recommandé. }\end{array}$ \\
\hline
\end{tabular}




\begin{tabular}{|c|c|c|}
\hline $\begin{array}{l}\text { Aspect et } \\
\text { dimensions }\end{array}$ & $\begin{array}{l}\text { Évaluation } \\
\text { (Accord } \\
\text { interjuge) }\end{array}$ & Justification \\
\hline \multicolumn{3}{|l|}{ INTERPRÉTER } \\
\hline $\begin{array}{l}\text { Prioriser les } \\
\text { données }\end{array}$ & $\begin{array}{l}\text { Accomplie } \\
\quad(2 / 3)\end{array}$ & $\begin{array}{l}\text { 1- Semble avoir bien réalisé que les vomissements et les nausées étaient des données } \\
\text { significatives. Ceci a aidé à valider le besoin d'insérer le tube NG. } \\
\text { 2- Capacité à faire les liens pertinents en débreffage. } \\
\text { 3- Simulation : examen clinique abdominal, mesure de constantes, repropose la bassine } \\
\text { pleine de vomi... retarde la pose de la sonde. }\end{array}$ \\
\hline $\begin{array}{l}\text { Comprendre les } \\
\text { données }\end{array}$ & $\begin{array}{l}\text { En } \\
\text { développement } \\
\quad(2 / 3)\end{array}$ & $\begin{array}{l}\text { 1- Je sentais de l'incertitude de la part de l'étudiante. Elle avait prédéterminé le besoin } \\
\text { d'insérer un tube NG et semblait « suivre une recette » relativement à l'intervention. } \\
\text { Elle ne semblait pas faire le lien entre la chirurgie abdominale et la possibilité d'un } \\
\text { iléus paralytique. } \\
\text { 2- Besoin d'aide pour établir la causalité (iléus paralytique postop). } \\
\text { 3- Débreffage : prise de conscience de la désorganisation, les liens entre les signes } \\
\text { cliniques, les vomissements et un probable iléus paralytique postopératoire ne sont } \\
\text { pas spontanément évoqués. Mais pendant la simulation l'intervention de poser le tube } \\
\text { est correcte. }\end{array}$ \\
\hline \multicolumn{3}{|l|}{ INTERVENIR } \\
\hline $\begin{array}{l}\text { Agir calmement } \\
\text { et avec confiance }\end{array}$ & $\begin{array}{l}\text { En } \\
\text { développement } \\
\quad(3 / 3)\end{array}$ & $\begin{array}{l}\text { 1- Je pense que l'étudiante commençait à explorer le rôle de leadership. Avait de la } \\
\text { difficulté à s'affirmer auprès de l'épouse au sujet du jus d'orange par exemple. Elle } \\
\text { admet un manque de confiance dans le débreffage, ce qui est commun dans les } \\
\text { circonstances et un excellent insight. } \\
\text { 2- Manque de confiance pour donner directives à la famille. } \\
\text { 3- Simulation : désorganisation jusqu'à la pose de la sonde. La présence de l'épouse, et } \\
\text { ses propositions (jus d'orange...) ne sont pas refusées et argumentées. Un peu de } \\
\text { passivité. }\end{array}$ \\
\hline $\begin{array}{l}\text { Communiquer } \\
\text { clairement }\end{array}$ & $\begin{array}{l}\text { Accomplie } \\
(3 / 3)\end{array}$ & $\begin{array}{l}\text { 1- C'était beau de voir que l'étudiante interagissait avec le client et faisait des efforts } \\
\text { évidents avec l'épouse. Elle expliquait bien tout ce qu'elle faisait avant de le faire et } \\
\text { donnait l'occasion au client de poser des questions. }\end{array}$ \\
\hline
\end{tabular}




\begin{tabular}{|c|c|c|}
\hline $\begin{array}{c}\text { Aspect et } \\
\text { dimensions }\end{array}$ & $\begin{array}{l}\text { Évaluation } \\
\text { (Accord } \\
\text { interjuge) }\end{array}$ & Justification \\
\hline & & $\begin{array}{l}\text { 2- Entre elles et avec la famille, pourrait être plus explicite. } \\
\text { 3- Simulation : explique ce qu'elles font, semblent sûres d'elles, de leurs gestes. }\end{array}$ \\
\hline $\begin{array}{l}\text { Intervenir de } \\
\text { façon planifiée et } \\
\text { avec flexibilité }\end{array}$ & $\begin{array}{l}\text { Accomplie } \\
(3 / 3)\end{array}$ & $\begin{array}{l}\text { 1- A bien surveillé le progrès lors des essais à insérer le tube NG. A essayé de changer } \\
\text { aussi. A bien expliqué au client comment signaler de la douleur ou une difficulté } \\
\text { 2- N'avait pas anticipé l'ajustement nécessaire aux besoins de la famille. } \\
\text { 3- Simulation : les interventions sont basées sur les données du patient objectives et } \\
\text { subjectives. } \\
\text { Débreffage : argumentation des interventions menées. }\end{array}$ \\
\hline $\begin{array}{l}\text { Faire preuve de } \\
\text { compétence }\end{array}$ & $\begin{array}{c}\text { Accomplie } \\
(3 / 3)\end{array}$ & $\begin{array}{l}\text { 1- Aucun commentaire. } \\
\text { 2- Nomme elle-même le besoin d'améliorer la rapidité. } \\
\text { 3- Simulation : manque de connaissances, semblent }{ }^{4} \text { être dans l'action sans développer } \\
\text { une réflexion approfondie. Ne communiquent pas assez. } \\
\text { Débreffage : les compétences théoriques sont formulées, mais n'ont pas été assez } \\
\text { visibles. }\end{array}$ \\
\hline \multicolumn{3}{|l|}{ RÉFLÉCHIR } \\
\hline $\begin{array}{l}\text { Évaluer et } \\
\text { s'autoévaluer }\end{array}$ & $\begin{array}{c}\text { Accomplie } \\
(3 / 3)\end{array}$ & $\begin{array}{l}\text { 1- A bien contribué au débreffage avec enthousiasme et authenticité. A bien analysé des } \\
\text { moments clés (p. ex. le jus d'orange, la présence de l'épouse, les imprévus). } \\
\text { 2- Démontré en débreffage. } \\
\text { 3- Débreffage : l'enseignante les aide beaucoup à verbaliser et évaluer leur } \\
\text { performance. Les solutions de rechange, par contre, viennent plus facilement. }\end{array}$ \\
\hline $\begin{array}{l}\text { Démontrer une } \\
\text { volonté de } \\
\text { s'améliorer }\end{array}$ & $\begin{array}{l}\text { Accomplie } \\
(2 / 3)\end{array}$ & $\begin{array}{l}\text { 1- A compris la valeur du débreffage et de sa réflexion en termes de sa future pratique. } \\
\text { A bien identifié, de façon concrète, des points à améliorer. « Être prête à agir et } \\
\text { penser avec les connaissances qu'on a » (même si on ne connaît pas tout). A bien } \\
\text { suivi, accepté et ajouté aux points partagés par la professeure. } \\
\text { 2- Voit peu les aspects positifs et les forces. } \\
\text { 3- Débreffage: motivation à s'inscrire dans un processus d'amélioration continue, } \\
\text { compare avec des expériences en stage, identifie les points à renforcer. }\end{array}$ \\
\hline
\end{tabular}

